PlastOx 2007 (2009) 45-55

(C) EDP Sciences, 2009

DOI: $10.1051 /$ ptox $/ 2009005$

\title{
Structures et défauts de structure des joints de grains
}

\section{Priester}

ICMPE/CNRS, 15 rue Georges Urbain, 94407 Vitry-sur-Seine, France

e-mail : louisette.priester@glvt-cnrs.fr

\begin{abstract}
Résumé. Pendant longtemps le joint de grains a été considéré comme une couche amorphe entre deux cristaux. Ce n'est qu'au milieu du $20^{\text {ème }}$ siècle, avec la découverte expérimentale de l'existence de propriétés vectorielles des joints de grains que l'idée d'ordre intergranulaire a émergé. L'hypothèse à la base de la recherche de la structure d'équilibre d'un joint de grains "idéal" est qu'une situation énergétiquement favorable doit être associée à l'existence d'un ordre commun aux deux cristaux. La recherche de cet ordre s'effectue à différents niveaux : macroscopique avec la bicristallographie, microscopique avec la description de la structure intergranulaire en termes de dislocations et enfin à l'échelle nanoscopique avec l'arrangement des atomes dans l'interface. Toute rupture de périodicité traduit l'existence d'un défaut dans la structure du joint de grains. Nous décrirons en particulier les dislocations extrinsèques qui sont pour le joint l'équivalent des dislocations dans la matrice ; elles proviennent généralement de l'interaction entre les dislocations des cristaux avec les joints de grains et sont responsables de la plasticité des interfaces.
\end{abstract}

\section{ORDRE GÉOMÉTRIQUE : BICRISTALLOGRAPHIE}

Deux cristaux de même nature reliés l'un à l'autre par une opération d'interface (rotation, déformation ou opération plus complexe) forment un bicristal. Un joint de grains est obtenu en introduisant un plan dans le bicristal et en rejetant les atomes de chacun des cristaux de part et d'autre de ce plan (Fig. 1).

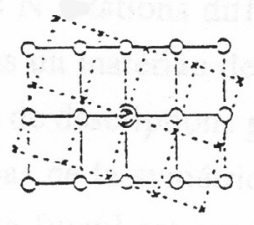

(a)

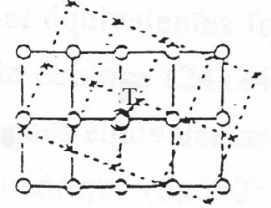

(b)

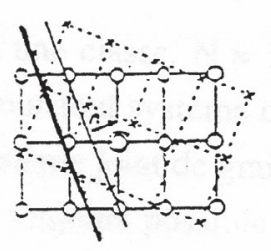

(c)

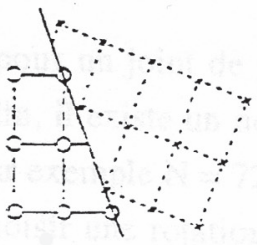

(d)

Figure 1. Schéma montrant les différentes opérations pour former un joint de grains : rotation (a) translation rigide d'un réseau par rapport à l'autre (opération d'interface) (b) - Orientation et position d'un plan de joint (c) - Rejet des atomes de chaque cristal d'un côté et de l'autre de ce plan (d).

Les paramètres géométriques ou degrés de liberté décrivant une interface peuvent être classés en deux catégories :

- 6 paramètres précisent l'opération d'interface : 3 pour la transformation, par exemple 1 angle et deux cosinus directeurs pour l'axe de la rotation $\mathbf{R}$, et 3 pour le vecteur de translation rigide $\mathbf{t}$

- 3 paramètres définissent le plan de l'interface : 2 pour son orientation, donnée par la normale $\mathbf{n}$ au plan, et 1 pour sa position précisée par un vecteur $\mathbf{d}$ sur la normale.

Une terminologie particulière s'applique aux joints de grains définis par une rotation $\theta$ [uvw] et par un plan $\{\mathrm{hkl}\}$ de normale $\mathbf{n}$ selon leurs caractéristiques :

$\mathbf{n} \perp[\mathrm{uvw}] \quad \Rightarrow \quad$ Joint de flexion

$\mathbf{n} / /[\mathrm{uvw}] \Rightarrow$ Joint de torsion

Article disponible sur le site http://plastox.edpsciences.org ou http://dx.doi.org/10.1051/ptox/2009005 
n incliné/[uvw]

$\Rightarrow \quad$ Joint mixte

$\{\mathrm{kkl}\}_{1} \equiv\{\mathrm{kkl}\}_{2}$

$\Rightarrow$ Joint symétrique

$\{\mathrm{kkl}\}_{1} \neq\{\mathrm{kkl}\}_{2}$

$\Rightarrow$ Joint asymétrique

n varie le long du joint $\Rightarrow$ Joint à facettes

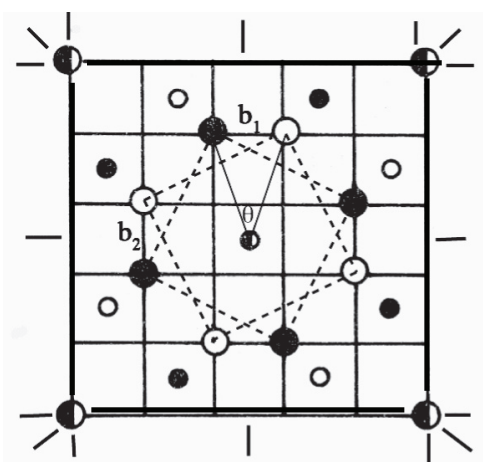

Figure 2. Maille de coïncidence d'un bicristal $\Sigma=5\left(\theta=36^{\circ} 9\langle 100\rangle\right)$ de structure cubique délimité par les nœuds bicolores (noir/blanc) et réseau DSC qui découpe les côtés de la maille de coïncidence en cinq; $\mathbf{b 1}$ et $\mathbf{b 2}$ sont les vecteurs élémentaires du réseau DSC.

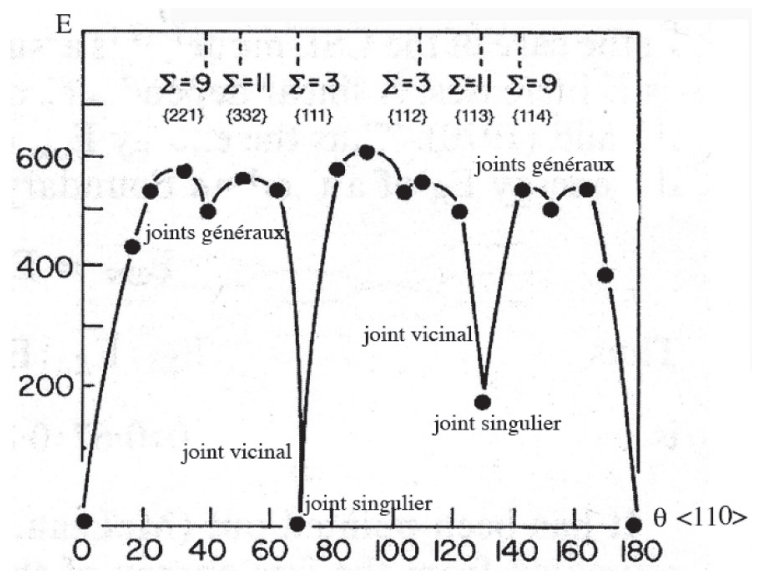

Figure 3. Evolution de l'énergie intergranulaire en fonction de la désorientation $\theta\langle 110\rangle$. Les positions de coïncidence sont repérées sur l'axe horizontal du haut. Quelques joints singuliers, vicinaux et généraux sont également marqués pour illustrer la classification des joints selon leur énergie.

\subsection{Réseau de coïncidence}

Le réseau de coïncidence dit "de sites de coïncidence" (CSL) est formé en considérant les nœuds communs aux réseaux des deux cristaux. Ce réseau est caractérisé par un indice de coïncidence $\Sigma$ égal au rapport entre le volume de la maille de coïncidence et le volume de la maille primitive du cristal. $\Sigma$ est aussi l'inverse de la densité de noeuds communs dans la maille de coïncidence (Fig. 2). C'est un entier, impair pour les matériaux cubiques. $\Sigma$ varie d'une manière discontinue avec $\theta$ et aucune relation biunivoque n'existe entre la valeur de $\Sigma$ et l'énergie intergranulaire (Fig. 3) [1-3].

La considération des énergies a donné lieu à une classification des joints de grains, similaire à celle des surfaces. Un joint singulier présente un minimum dans la courbe qui relie l'énergie des joints à un 
au moins des paramètres macroscopiques. Il est vicinal si son énergie est voisine de celle d'un joint singulier. Les joints généraux ont des énergies situées aux maxima d'énergie et qui varient peu avec le paramètre géométrique considéré. Dans cette définition, un joint de grains peut être singulier par rapport à un degré de liberté et vicinal ou général par rapport à un autre.

\subsection{Réseau DSC}

Le sigle DSC provient de la terminologie anglaise originale "Displacement Shift Complete". Plus récemment et plus explicitement; on l'associe à "Displacement Symmetry Conserving ; en effet, une translation du réseau de coïncidence égale à un vecteur du réseau DSC laisse le réseau CSL inchangé (Fig. 2). Le réseau DSC est le réseau de translation du bicristal qui définit les vecteurs de Burgers des dislocations parfaites des joints de grains [1].

\subsection{Réseau 0}

Le réseau 0 est le lieu de tous les points en bon accord entre le cristal 1 et le cristal 2 séparés par des régions de mauvais accord. Il rend donc compte de l'invariant par rapport au monocristal. Les sites du réseau 0 , contrairement à ceux du réseau CSL, peuvent avoir des coordonnées internes aux mailles des cristaux. En conséquence, le réseau 0 varie continûment avec la désorientation q autour d'un axe de rotation donné. Un point appartient au réseau 0 s'il reste invariant dans la transformation qui relie le cristal 1 au cristal 2. Dans l'approximation de Bollmann (translation rigide nulle), l'équation fondamentale du réseau 0 est [1] :

$$
\left(\mathbf{I}-\mathbf{R}^{-1}\right) \mathbf{x}_{0}=\mathbf{B}_{\mathbf{m}}=\Sigma \mathbf{b}_{\mathbf{m}}
$$

I est la matrice Identité, $\mathbf{R}$ est la matrice de rotation et $\mathbf{x}_{0}$ est un vecteur qui repère un point 0 dans le plan du joint. $\mathbf{B}_{\mathbf{m}}$ est un vecteur de translation du réseau et peut donc toujours être considéré comme la somme de vecteurs de Burgers de la matrice $\mathbf{b}_{\mathbf{m}}$.

\subsection{Réseau 02}

Un joint de grains à grand angle $\theta$ [uvw] est le plus souvent écarté d'une désorientation de coïncidence d'un angle $\Delta \theta$ autour d'un axe [u'v'w']. Considérant que le réseau de coïncidence est un second invariant, Bollmann introduit le réseau 02. De la même manière que le réseau 0 précise les sites invariants entre les réseaux de translation des cristaux désorientés de $\theta$ [uvw], le réseau 02 précise le sites invariants entre les réseaux DSC de translation du bicristal tournés de $\Delta \theta$ [u'v'w'].

L'équation fondamentale du réseau 02 (2) est similaire à l'équation (1) :

$$
\left(\mathbf{I}-\mathbf{D}^{-1}\right) \mathbf{x}_{02}=\Sigma \mathbf{b}_{\mathbf{D S C}}
$$

$\mathbf{D}$ est la matrice écart entre celle décrivant la rotation réelle des cristaux $\mathbf{R}$ et celle de la coïncidence la plus proche $\mathbf{R}_{\mathrm{c}}\left(\mathbf{D}=\mathbf{R} \mathbf{R}_{\mathrm{c}}^{-1}\right)$. Le réseau 02 existe toujours et varie continûment avec l'écart angulaire $\Delta \theta \mathrm{g}$ la désorientation de coïncidence.

Le formalisme de Bollmann a été développé pour prédire des relations d'orientation préférentielles entre deux cristaux et non le plan préférentiel de l'interface qui seul conduit à une faible énergie interfaciale. Une relation particulière entre cristaux n'implique pas nécessairement un plan particulier et réciproquement. Plusieurs critères ont été avancés pour prédire le plan de l'interface : grande densité de sites de coïncidence dans ce plan, grande distance interplanaire, mais aucun d'eux n'est satisfaisant. 


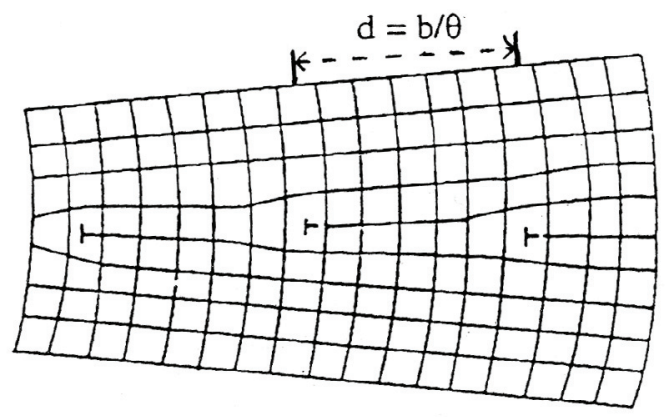

Figure 4. Modèle de Read et Shockley pour les joints de grains de flexion de faible angle.

\section{ORDRE DES CONTRAINTES : DISLOCATIONS INTRINSÈQUES DES JOINTS DE GRAINS}

\subsection{Modèle de Read et Shockley}

L'interaction élastique entre des dislocations coin de même signe situées dans les plans de glissement parallèles et équidistants conduit à la formation d'une paroi de dislocations et à une désorientation de part et d'autre de cette paroi telle que $\theta=\mathrm{b} / \mathrm{d}$ (Fig. 4). La paroi ainsi constituée est le premier modèle d'un joint de flexion de faible angle. Son énergie est la somme des énergies des dislocations présentes par unité de surface du joint, selon la formule classique de Read et Shockley :

$$
\gamma_{\mathrm{q}}=\gamma_{0} \theta(\mathrm{A}-\ln \theta)
$$

avec $\gamma_{0}$ et $\mathrm{A}$ des constantes Fondamentalement, ce modèle est à la base de toutes les descriptions des interfaces en termes de dislocations $[2,3]$.

\subsection{Circuit et formule de Frank}

La densité de vecteur de Burgers dans le joint de grains, nécessaire pour réaliser la compatibilité à l'interface entre les deux réseaux, peut être déterminée à partir du circuit de Frank (Fig. 5) analogue au circuit de Burgers pour les dislocations. Un défaut de fermeture $\mathbf{B}$ apparaît tel que :

$$
\mathbf{B}=\left(\mathbf{I}-\mathbf{R}^{-1}\right) \mathbf{p}
$$

avec $\mathbf{p}\left(\mathbf{X}_{2}\right.$ sur la figure 5) un vecteur non négligeable dans le plan du joint.

A ce stade B n'est pas discrétisé, il n'est pas considéré comme la somme de vecteurs de Burgers de la matrice ou du réseau DSC. Une telle discrétisation dépend de processus de relaxation qui ne peuvent pas être prédits par des considérations géométriques.

\subsection{Modèle de Bollmann}

Dans le modèle de Bollmann [1], l'interface est constituée de régions en bon accord définies par les deux invariants (monocristal et CSL) entre lesquelles se situent des régions en désaccord où se localisent des dislocations dites "structurales" ou "intrinsèques" [1]. Ces dislocations rendent totalement compte de la désorientation entre les deux cristaux. Dans la formule (4) le vecteur B est maintenant discrétisé : $\mathbf{B}=\sum \mathbf{n}_{\mathbf{i}} \mathbf{b}_{\mathbf{i}}$ avec $\mathbf{b}_{\mathbf{i}}$ le vecteur de Burgers des dislocations intrinsèques et $\mathbf{n}_{\mathbf{i}}$, le nombre de dislocations intrinsèques coupées par le vecteur $\mathbf{p}$ dans le plan du joint de grains.

Cette discrétisation doit être vue comme une premier mode de relaxation linéaire des contraintes d'incompatibilité. Elle permet de proposer une structure d'équilibre possible de l'interface en sachant 


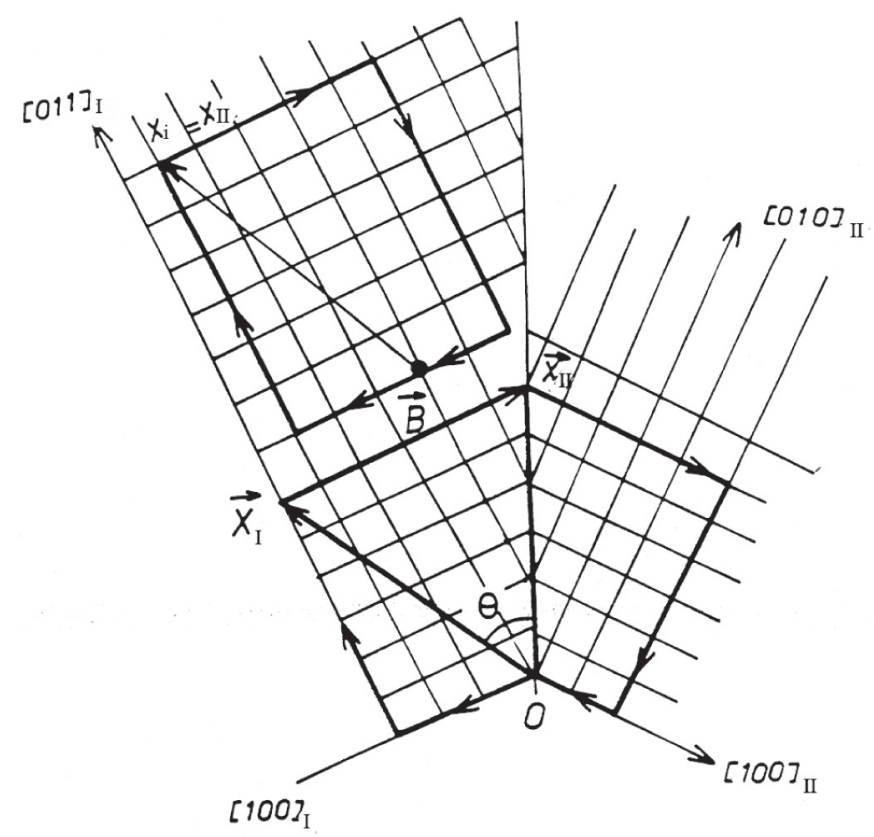

Figure 5. Circuit de Frank pour définir le défaut de fermeture associé à la déformation dans le joint.

que seules des considérations physiques décident de la structure réelle [3]. Les dislocations intrinsèques (intrinsèque $\equiv$ équilibre) sont de deux types :

- Les dislocations intrinsèques primaires compensent la déviation $\theta$ [uvw] par rapport au monocristal, leur périodicité est donc celle du réseau 0 (Fig. 6). Leurs vecteurs de Burgers sont ceux des dislocations de matrice. Pour un joint de flexion, le réseau est constitué de dislocations coins parallèles à l'axe de rotation. Dans le cas d'un joint de torsion, il s'agit d'une grille de dislocations de caractère vis. Dans tous les cas, la distance de ces dislocations est donnée par l'expression :

$$
\mathrm{d}=\frac{\left|\mathrm{b}_{\mathrm{m}}\right|}{2 \sin \theta / 2}
$$

Lorsque l'angle de désorientation entre les cristaux augmente, la distance entre dislocations devient si petite qu'elle n'est plus compatible avec la périodicité du réseau du cristal. La notion de dislocations discrètes de vecteur $b_{m} n$ 'a plus de signification physique. Pour décrire l'interface, on fait alors appel au second invariant, le réseau CSL.

- Les dislocations intrinsèques secondaires rendent compte de l'écart angulaire $\Delta \theta$ [u'v'w'] du joint réel par rapport à la désorientation de coïncidence la plus proche. Leur périodicité est celle du réseau 02 et leurs vecteurs de Burgers sont des vecteurs du réseau DSC.

Dans ce modèle, les joints à faible angle et coïncidents sont uniquement décrits par des dislocations primaires. Dans les joints proches d'une position de coïncidence, il faut introduire des dislocations secondaires pour compenser l'écart entre le joint de coïncidence et le joint réel. Dans les joints généraux, les cœurs des dislocations intrinsèques se chevauchent, celles-ci n'ont plus de signification physique.

\section{ORDRE ATOMIQUE : MODÈLE DES UNITÉS STRUCTURALES}

Tout d'abord prédit par des considérations géométriques, l'arrangement des atomes en unités structurales dans le joint a été déduit des simulations numériques et confirmé par les observations 


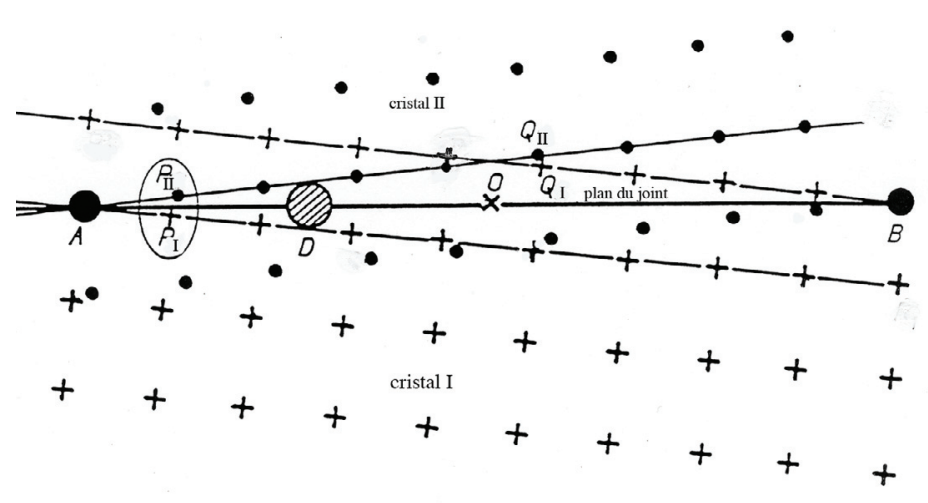

Figure 6. Schéma montrant la localisation des dislocations primaires dans un joint de flexion de faible angle. A, O et $\mathrm{B}$ sont des points $0 . \mathrm{P}_{1}$ et $\mathrm{P}_{2}$. $\left(\mathrm{Q}_{1}\right.$ et $\left.\mathrm{Q}_{2}\right)$ sont des nœuds homologues assimilés à un seul nœud. $\mathrm{D}$ est le site de plus mauvais accord dans le plan du joint, là où se situe une dislocation primaire $\mathrm{D}$.

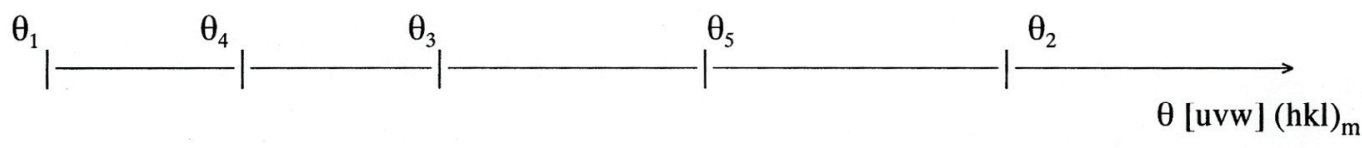

AAAA... joint singulier favorisé
ABAB... joint singulier
BBBB....

joint singulier

favorisé
AAABAAAB...
ABBBBABBBB...
joint général

Figure 7. Description des joints de grains en termes d'unités structurales.

en microscopie électronique à haute résolution (METHR). Les simulations ont précisé la largeur du joint, environ deux distances interplanaires, son énergie minimale et la configuration atomique la plus stable associée.

\subsection{Principe du modèle des unités structurales et limites de la description}

Tout joint est constitué d'un nombre limité d'unités structurales (polyèdres d'atomes) disposés périodiquement. Ces polyèdres sont équivalents aux mailles des cristaux mais avec des formes plus complexes que le cube, l'hexagone... On distingue :

- Les joints favorisés constitués d'un seul type d'unités, représentés par exemple par "AAAAA...". Parmi les joints coïncidents, seuls ceux de plus faible énergie sont favorisés $(\Sigma 3\{111\}$ et $(\Sigma 11\{113\}$ sur la figure 3).

- Les joints coüncidents non favorisés formés par une séquence simple de deux unités structurales, par exemple "ABABAB..."

- Les joints généraux intermédiaires présentant une séquence complexe d'unités structurales.

Principe de construction d'un joint : Tout joint de grains de longue période et de désorientation q [uvw] peut être décrit par un arrangement séquentiel d'unités structurales associées à deux joints délimitants $\mathrm{q}_{1}$ et $\square_{2}$ de courte période possédant le même axe de rotation et le même plan médian (Fig. 7) [2, 3]. 


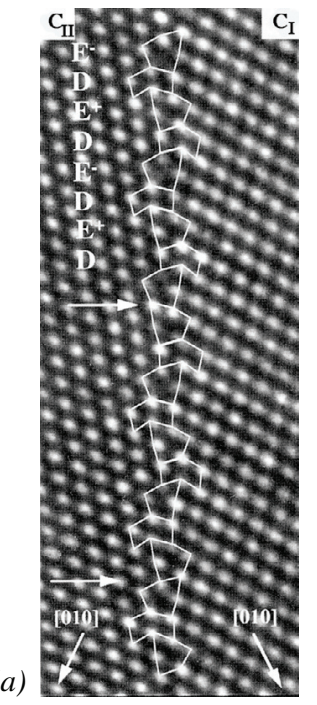

(b)

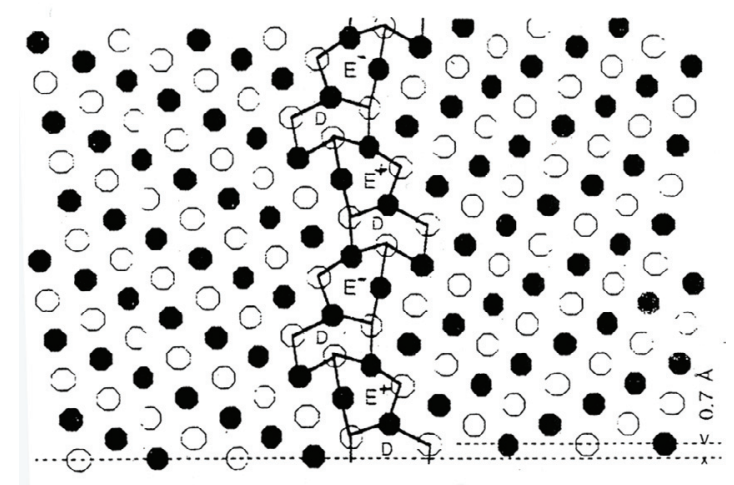

Figure 8. Structures, observée (a) et simulée (b) - noter le très bon accord- du joint $\Sigma 11\{332\}$ dans le nickel. Les flèches blanches indiquent la présence de dislocations extrinsèques (voir texte plus loin [4].

La figure 8 présente les images, en METHR et simulée, d'un joint général $\Sigma 11\{332\}$ dans le nickel : sa période est constituée de deux unités D du joint $\Sigma 3$ et de deux unités E du joint $\Sigma 9\{221\}$, symétriques l'une de l'autre par rapport au plan du joint [3,4].

Limites : l'unité d'un joint favorisé peut être distordue dans un joint intermédiaire dont la désorientation est trop éloignée de la référence, par exemple l'unité B dans le joint général $\theta_{4}$ (Fig. 7). L'utilisation d'un autre joint délimitant dit du second ordre, par exemple $\theta_{3}$, constitué d'une séquence simple d'unités C (regroupement de A et B) permet de décrire le joint $\theta_{4}$ comme "AACAAC...". Par ailleurs, dans un joint favorisé, l'unité structurale peut prendre différentes configurations d'énergies voisines. Soit a et b les nombres d'unités dans deux joints favorisés. Tout joint intermédiaire peut alors être décrit en principe par différentes combinaisons $\mathrm{N}$ des unités favorisées : $\mathrm{N}=\mathrm{a}^{\mathrm{n}} \mathrm{b}^{\mathrm{m}}$. Dans tous les cas, seule l'observation expérimentale peut préciser la structure réellement adoptée par le joint.

Le modèle des unités structurales n'a un caractère réellement prédictif que pour les joints de flexion symétriques autour d'axes simples. La description des joints de torsion est complexe, elle nécessite l'introduction d'unités dites “filler", non prévues, pour combler des vides entre les unités prédites par le modèle. Quelques constructions de joints asymétriques en termes d'unités sont proposées depuis peu.

\subsection{Le modèle « Unités structurales / dislocations intrinsèques »}

Une corrélation étroite a été établie entre le modèle des unités structurales et celui des dislocations intrinsèques. Chaque unité minoritaire $B$ peut être considérée comme le cœur d'une dislocation secondaire dont le vecteur de Burgers est un vecteur du réseau DSC du joint délimitant constitué d'unités A (Fig. 9).

En conclusion des considérations sur l'ordre, les descriptions des joints de grains décrites ci-dessus concernent leur structure d'équilibre, mais les joints réels, à l'instar des cristaux, ne sont jamais parfaits. Ce sont les imperfections qui confèrent aux joints de grains la plupart de leurs caractéristiques d'emploi : résistance ou non à la déformation, à la corrosion, à la fracture et certaines de leurs propriétés électroniques... Les défauts présentés sont formellement divisés en deux catégories : défauts structuraux, décrits ci-dessous, et défauts chimiques, objets d'une seconde partie du cours. En réalité, les deux types de défauts sont généralement associés. 


$\begin{array}{ll}\mathrm{A} & \perp \\ \mathrm{A} & \perp \\ \mathrm{A} & \perp \\ \mathrm{A} & \perp \\ \mathrm{B} & \perp \\ \mathrm{A} & \perp \\ \mathrm{A} & \perp \\ \mathrm{A} & \perp \\ \mathrm{A} & \perp \\ \text { B } & \perp \\ \mathrm{A} & \perp \\ \mathrm{A} & \perp\end{array}$

Figure 9. Corrélation entre la description de la structure d'un joint de grains en termes de dislocations intrinsèques ( $\perp$ dislocations primaires et $\perp$ secondaires) et sa description en termes d'unités structurales $\mathbf{A}$ et $\mathbf{B}$ [3].

\section{DÉFAUTS DE STRUCTURE DES JOINTS DE GRAINS : DISLOCATIONS EXTRINSÈQUES}

\subsection{Origine et caractéristiques des dislocations extrinsèques}

Du latin "extrinsecus" signifiant "de l'extérieur", ce terme appliqué à une dislocation intergranulaire peut être interprété de deux manières :

- Soit l'accent est mis sur l'origine de la dislocation : la dislocation provient de l'extérieur du joint, le plus souvent d'un des cristaux avoisinants, le joint jouant un rôle de puits pour les dislocations de matrice.

- Soit l'accent est mis sur la structure fautée du joint : extrinsèque signifie alors "en dehors de la structure d'équilibre du joint de grains", quelle que soit l'origine de la dislocation.

En termes élastiques, une dislocation extrinsèque détruit la périodicité de l'arrangement des dislocations intrinsèques (Fig. 10) et induit un champ de contraintes élastiques à longue distance. Son vecteur de Burgers n'entre pas dans la densité de vecteurs de Burgers $\mathbf{B}$ donnée par l'équation (4) pour une relation fixe entre cristaux :

$$
\mathbf{B}+\mathbf{b}_{\mathbf{e}}=\mathbf{B}^{\prime}
$$

B' ne rend pas compte de la désorientation globale entre les cristaux, seul un changement extrêmement localisé de la désorientation se produit à l'aplomb de la dislocation.

En termes de cour, une dislocation extrinsèque correspond à une rupture de périodicité de l'arrangement des unités structurales, provoquée par un manque ou un surplus d'une (ou plusieurs) de ces unités. La figure 10 précise le lien étroit entre la description d'une dislocation extrinsèque par référence au réseau de dislocations intrinsèques et sa description en termes d'unités structurales ; un exemple en est donné pour le joint $\Sigma 11\{332\}$ du nickel (Fig. 8a) [3].

Les vecteurs de Burgers des dislocations extrinsèques parfaites sont des vecteurs de translation du joint de grains. Pour un joint de grains donné, ils sont égaux aux vecteurs, non nécessairement élémentaires, du réseau DSC du joint de coïncidence le plus proche $: \mathbf{b}_{\mathbf{e}}=\mathbf{b}_{\text {DSC }}$ ou $\mathbf{b}_{\mathbf{e}}=\Sigma \mathbf{b}_{\text {DSC }}$. 


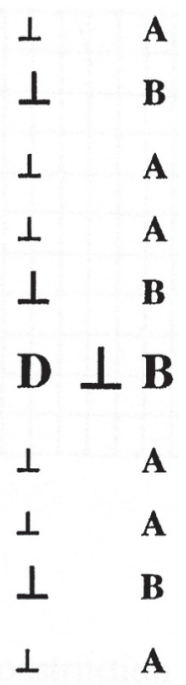

Figure 10. Une dislocation extrinsèque $\mathbf{D}(\perp)$ introduit une rupture de périodicité dans la structure d'un joint de grains décrite en termes de dislocations intrinsèques primaires $(\perp)$ et secondaires $(\perp)$ ou en termes d'unités structurales $\mathbf{A}$ et $\mathbf{B}$. La dislocation correspond à une unité $\mathbf{B}$ (ou $\mathbf{A}$ ) en plus (ou moins). Sur la figure 8a, une unité $\mathrm{D}$ manque dans la structure d'équilibre du joint de période EDED à deux endroits (flèches blanches) indiquant la présence de deux dislocations extrinsèques.

\subsection{Formation et accommodation des dislocations extrinsèques [3]}

La formation d'une dislocation extrinsèque requiert généralement une interaction entre une dislocation de matrice et un joint de grains. Elle peut résulter du transfert d'une dislocation de matrice d'un cristal à l'autre laissant dans le joint un produit. Elle peut aussi provenir de la décomposition d'une dislocation de matrice piégée dans le joint en dislocations dont les vecteurs de Burgers sont ceux du réseau DSC (Fig. 11). Ces réactions sont difficiles à basse température, les joints jouant le rôle d'obstacles forts à la déformation.

Quelle que soit la température, l'entrée d'une dislocation dans un joint nécessite de fortes contraintes. Elle peut intervenir au début de la déformation plastique, mais dès que les joints sont contraints, les empilements de dislocations d'un côté du joint génèrent des sources de dislocations dans le joint opposé (ou du même côté). Ce mode de transmission de la contrainte dit "indirect" dépend peu de la structure du joint et implique les régions proches du joint qui sont dans un état avancé de déformation. Un transfert "direct" de la dislocation d'un grain à l'autre au travers du joint requiert que trois conditions soient remplies : (i) l'angle entre les traces des plans de glissement dans le plan du joint doit être le plus petit possible; (ii) le vecteur de Burgers de la dislocation extrinsèque (vecteur du réseau DSC), produite par la réaction et restant dans le joint, doit être aussi petit que possible ; (iii) la contrainte sur la dislocation émise dans le cristal doit être très élevée. Ces restrictions entraînent que le transfert direct est rare.

Si la température est suffisamment élevée, les contraintes associées aux premières dislocations entrant dans un joint peuvent relaxer, permettant à ce dernier d'absorber d'autres dislocations. Les processus de relaxation : incorporation de la dislocation dans la structure intergranulaire ou délocalisation de la contrainte dans le joint dépendent de la structure fine du joint de grains.

- Le modèle d'incorporation a été établi pour des joints de flexion périodiques et pour des dislocations extrinsèques parallèles au réseau intrinsèque. Chaque dislocation piégée dans le joint se décompose en produits glissiles et sessiles, conférant au joint un caractère hors d'équilibre. Les produits glissiles 


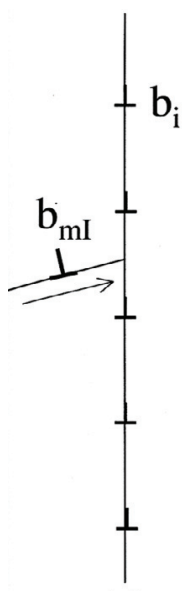

(a)

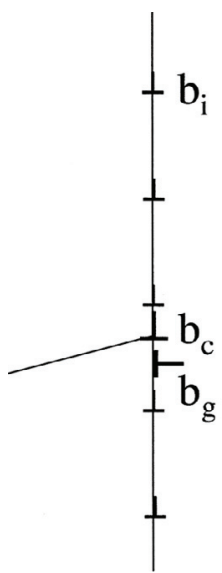

(b)

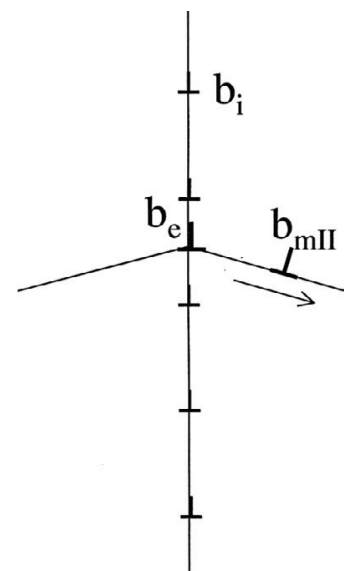

(c)

Figure 11. Schémas montrant l'interaction d'une dislocation de matrice avec un joint de grains : (a) avant réaction ; b) décomposition d'une dislocation de matrice en plusieurs dislocations extrinsèques ; (c) transmission de la dislocation de matrice d'un cristal à l'autre avec résidu dans le joint.

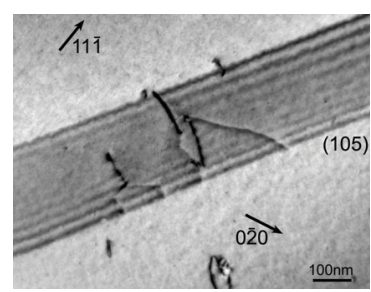

$\Sigma=3-\mathrm{T}_{\mathrm{amb}} \mathrm{t}=0$

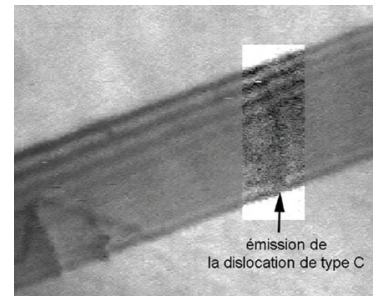

$\Sigma=3-0,5 \mathrm{~T}_{\mathrm{F}} \mathrm{t}=1 \mathrm{~h}$

(a)

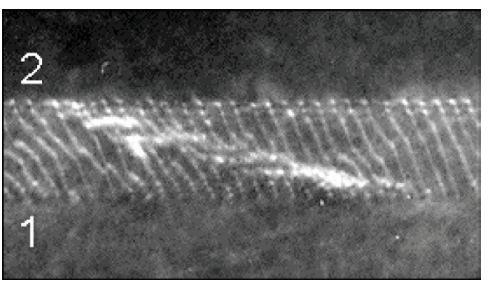

$\Sigma=9-\mathrm{T}_{\mathrm{amb}} \mathrm{t}=0$

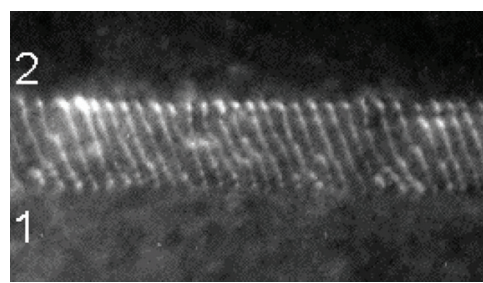

$\Sigma=9-\mathrm{T}_{\mathrm{amb}} \mathrm{t}=2$ jours

(b)

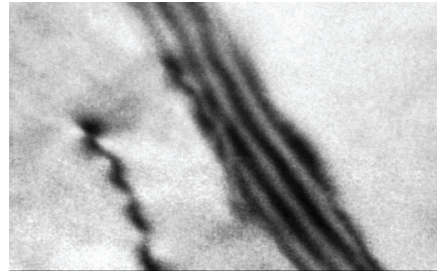

Joint général $-T_{a m b} t=0$

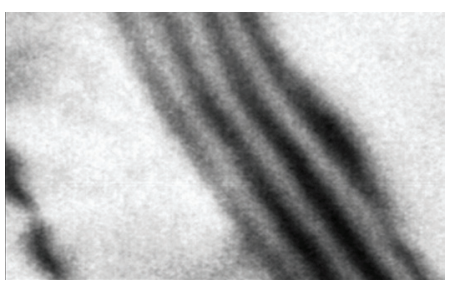

Joint général $-\mathrm{T}_{\mathrm{amb}} \mathrm{t}=\varepsilon$

(c)

Figure 12. (a) Les dislocations extrinsèques formées dans un joint de grains singulier $\Sigma=3$ à température ambiante évoluent peu sous l'effet d'une activation thermique : le joint est "dur", il conserve des contraintes à grande distance même après un traitement de 1 heure à $0,5 \mathrm{~T}_{\mathrm{F}}$; (b) Les produits extrinsèques formés dés l'entrée d'une dislocation de matrice dans un joint vicinal $\Sigma=9$ réagissent avec les produits intrinsèques pour former un nouveau réseau périodique d'équilibre après deux jours à température ambiante ; (c) la dislocation en tête d'un empilement dans un cristal entre dans un joint général dans lequel son champ de contrainte se disperse instantanément $(t=\varepsilon)$ : le joint est "mou" [5]. 
se déplacent rapidement vers les extrémités du joint, les produits sessiles se réarrangent avec les dislocations intrinsèques d'origine pour former un nouveau joint périodique dont la désorientation est modifiée. Dans ce processus, les dislocations extrinsèques deviennent intrinsèques.

- Le modèle de délocalisation considère que le cœur de la dislocation extrinsèque, localisé juste après l'entrée de la dislocation de matrice dans le joint, s'étend sous l'effet d'une activation thermique. Aucun produit discret n'apparaît, ce qui est formellement équivalent à la décomposition de la dislocation extrinsèque en un nombre très élevé de dislocations dont les vecteurs de Burgers sont infinitésimaux. Ce modèle semble favorisé dans les joints à caractère pseudo périodique, voire non périodique.

Ces modèles ont été confrontés aux phénomènes réellement observés en microscopie électronique en transmission, en prenant la précaution de coupler différentes techniques et échelles d'observation (microscopie conventionnelle, faisceau faible, in situ et haute résolution). La distinction entre joints (singulier, vicinal et général) basée sur l'énergie intergranulaire se révèle vite insuffisante pour distinguer les mécanismes opérationnels dans un joint donné ; seul le degré de périodicité dans le plan du joint semble jouer un rôle dans les réactions "dislocations/joints" ainsi que dans les processus et la cinétique de relaxation des contraintes intergranulaires. Ces comportements permettent de classer les joints de grains en deux grandes catégories :

- Les joints durs dans lesquels l'entrée des dislocations de matrice est difficile ; si elle advient, la relaxation des dislocations extrinsèques se fait lentement et n'est jamais complète, même à des températures de l'ordre de $0,8 \mathrm{~T}_{\mathrm{F}}\left(\mathrm{T}_{\mathrm{F}}\right.$ : température de fusion). Ce sont essentiellement les macles $\Sigma 3\{111\}$ et quelques joints singuliers et/ou de courte période.

- Les joints mous regroupent les joints généraux et les joints vicinaux d'écart à la coïncidence non négligeable.

La figure 12 illustre bien les différentes évolutions sous l'effet de la température et en fonction du temps des dislocations extrinsèques dans trois types de joints de grains du cuivre : un joint singulier extrêmement proche de la macle $\Sigma 3\{111\}$, un joint vicinal proche de $\Sigma 9\{221\}$ et un joint général [5].

\section{Références}

[1] Bollmann W., "Crystal defects and crystalline interfaces” (Springer Verlag, Berlin 1970).

[2] Sutton A.P. and Balluffi R.W., "Interfaces in Crystalline Materials" (Clarendon Press, Oxford, 1995).

[3] Priester L., “Les joints de grains - De la Théorie à l'Ingénierie” Les Editions de Physique (2006).

[4] Hardouin Duparc O., Poulat S., Larère A., Thibault J. and Priester L., Phil. Mag. A 80 (2000) 853.

[5] Couzinié J.P., Pettinari Strumel F. Décamps B. and Priester L., Proceedings of the International Conference Copper'06 : Better Properties for Innovative Products, Editor : Welter J.M., France (2006) pp. 283-287. 
